\title{
Vortex structures in mesoscopic superconducting spheres
}

\author{
B. J. Baelus, D. Sun, and F. M. Peeters* \\ Departement Fysica, Universiteit Antwerpen, Groenenborgerlaan 171, B-2020 Antwerpen, Belgium \\ (Received 7 October 2006; revised manuscript received 3 March 2007; published 29 May 2007)
}

\begin{abstract}
Vortex states in mesoscopic superconducting spheres are investigated within the framework of the phenomenological Ginzburg-Landau theory in the presence of an externally applied magnetic field. We treat the linearized first Ginzburg-Landau equation as an eigenvalue problem and find the cylindrical symmetric giant vortex states. Taking linear combinations of solutions of the linearized equation and minimizing the free energy, we obtain the stable multivortex and giant vortex states.
\end{abstract}

DOI: 10.1103/PhysRevB.75.174523

PACS number(s): 74.20.De, 74.78.Na

\section{INTRODUCTION}

Recent progress in nanofabrication and nanotechnology made it possible to study the properties of mesoscopic superconductors. A mesoscopic sample has sizes comparable to the coherence length $\xi$ and/or penetration depth $\lambda$, and their magnetic properties are highly affected by the boundary of the sample. Stable vortex configurations result from the competition between the triangular Abrikosov vortex lattice, having the lowest energy in bulk superconductors, and the boundary that tries to impose its symmetry.

Most of the previous studies on mesoscopic superconductors were restricted to thin superconducting samples [which can be considered as (quasi) two dimensional]. Circular mesoscopic disks have been the most popular in this respect, both theoretically ${ }^{1-6}$ and experimentally. ${ }^{7-13}$ Two kinds of fundamentally new vortex states were predicted theoretically: (i) multivortex states with a spatial arrangement of singly quantized vortex states (which can be considered as the mesoscopic analogon of the Abrikosov vortex state in bulk superconductors) and (ii) axially symmetric giant vortex states (or multiply quantized vortex states) with a single core in the center. When the size of the disk is large enough, also a combination of the two states can appear. ${ }^{5}$ Recently, experimental evidence for the existence of the two kinds of vortex states have been found by using the multiplesmall-tunnel-junction method. ${ }^{12}$ The effect of the geometry of the sample on the superconductivity was thoroughly investigated for thin mesoscopic samples (see, e.g., Refs. 7 and 14-21). It was found that it can lead to a new vortex configuration consisting of a single antivortex surrounded by vortices. ${ }^{14,22,23}$

In thin superconducting samples with thickness $d \ll \lambda, \xi$, which are uniform in the direction of the magnetic field, let us say the $z$ direction, one can assume that inside the sample the order parameter and the vector potential are independent of $z$. The same is true for the opposite limit of $d \rightarrow \infty$, e.g., superconducting cylinders. ${ }^{24}$ In both cases, vortices are straight lines inside the superconducting sample. But what happens when the sample is no longer uniform in the $z$ direction? In this case, we may expect that the vortices will bend due to the effect of the boundary. In the present paper, we consider strongly type-II mesoscopic superconducting spheres placed in an externally applied magnetic field. Solving the first Ginzburg-Landau equation, we calculate the (meta-)stable vortex states in such a sphere for different sphere radii. The stability and the properties of the giant and multivortex states are investigated for different values of the radius.

Recently, hollow superconducting spheres or spherical shells were studied by Du and Ju using the Ginzburg-Landau theory. ${ }^{25}$ The stable vortex configurations and the nucleation and/or splitting of vortex pairs near the equator were investigated. In spherical shells, vortices only nucleate at the boundary. In order to study vortex configurations inside a three-dimensional sample, one has to consider massive superconductors. In a previous work, ${ }^{26}$ the vortex structure in a mesoscopic superconducting wire containing a constriction was investigated. Breakup of the vortex structure near the constriction was found, where (i) curved vortices are formed, which leave the superconductor at the constriction, and (ii) the breakup of a giant vortex into smaller and/or individual vortices near the constriction. To our knowledge, vortex matter in superconducting spheres have not been studied up to now.

This paper is organized as follows. In Sec. II, we solve the linearized first Ginzburg-Landau equation by treating it as an eigenvalue problem and restricting ourselves to cylindrical symmetric states. In this way, we obtain the giant vortex states in superconducting spheres of different sizes. In Sec. III, we consider linear combinations of two different giant vortex states in order to find the possible multivortex states and the stability region of the giant vortex states. Finally, in Sec. IV, we summarize our results.

\section{GIANT VORTEX STATES}

Giant vortex states have cylindrical symmetry in samples of circular symmetry. To find the (meta-)stable giant vortex states in a sphere, we first solve the linearized first GinzburgLandau equation. This is expected to be a good approximation near the signal-to-noise ratio $(\mathrm{S} / \mathrm{N})$ boundary where $|\psi|^{2} \ll 1$ and, correspondingly, we may neglect the nonlinear term in the Ginzburg-Landan (GL) equation. The linearized first GL equation becomes, in cylindrical coordinates,

$$
\begin{aligned}
& \frac{1}{\rho} \frac{\partial}{\partial \rho}\left(\rho \frac{\partial \Psi}{\partial \rho}\right)+\frac{1}{\rho^{2}} \frac{\partial^{2} \Psi}{\partial \varphi^{2}}-2 i s \frac{\partial \Psi}{\partial \varphi}-s^{2} \rho^{2} \Psi+\frac{\partial^{2} \Psi}{\partial z^{2}} \\
& \quad=-\frac{2 m}{\hbar^{2}}(-\alpha) \Psi,
\end{aligned}
$$

where $s=e H / \hbar c, H=H / H_{c 2}$, and $\rho=\rho / \xi$. Since 


$$
-\alpha(T) \equiv \frac{\hbar^{2}}{2 m \xi^{2}}\left(1-\frac{T}{T_{c}}\right)
$$

we can write

$$
\begin{aligned}
& \frac{1}{\rho} \frac{\partial}{\partial \rho}\left(\rho \frac{\partial \Psi}{\partial \rho}\right)+\frac{1}{\rho^{2}} \frac{\partial^{2} \Psi}{\partial \varphi^{2}}-2 i s \frac{\partial \Psi}{\partial \varphi}-s^{2} \rho^{2} \Psi+\frac{\partial^{2} \Psi}{\partial z^{2}} \\
& \quad=-\left(1-\frac{T}{T_{c}}\right) \Psi .
\end{aligned}
$$

Giant vortex states are cylindrically symmetric, and hence, their order parameter should be cylindrically symmetric too, i.e.,

$$
\Psi(\rho, \varphi, z)=e^{i L \varphi} \psi(\rho, z) .
$$

In this case, Eq. (3) reduces to the following eigenvalue problem (with $\varepsilon=1-T / T_{c}$ ):

$$
-\frac{\partial^{2} \psi}{\partial \rho^{2}}-\frac{\partial^{2} \psi}{\partial z^{2}}-\frac{1}{\rho} \frac{\partial \psi}{\partial \rho}+\left(\frac{L^{2}}{\rho^{2}}+\frac{H^{2} \rho^{2}}{4}-H L\right) \psi=\varepsilon \psi
$$

Solving this differential equation with the boundary condition $\partial \psi / \partial r=0$ at the boundary $\sqrt{\rho^{2}+z^{2}}=R$ gives us the values of $\varepsilon$ and the order parameter. To follow the notation of Refs. 1 and 27 , we use the $\hat{L}$ operator, given by

$$
\hat{L}=-\frac{\partial^{2}}{\partial \rho^{2}}-\frac{\partial^{2}}{\partial z^{2}}-\frac{1}{\rho} \frac{\partial}{\partial \rho}+\left(\frac{L^{2}}{\rho^{2}}+\frac{H^{2} \rho^{2}}{4}-H L\right)-1,
$$

and the eigenvalues and eigenfunctions of the $\hat{L}$ operator can be found from the equation

$$
\hat{L} \psi_{L, n}(\rho, z)=\Lambda_{L, n} \psi_{L, n}(\rho, z),
$$

where $\Lambda=\varepsilon-1$ for fixed vorticity and radius, $L$ is the vorticity, and $n=1,2, \ldots$, enumerates the different states for the same vorticity $L . n-1$ gives also the number of nodes in the radial direction. The two-dimensional second-order differential equation is solved numerically using the finite difference technique.

The superconducting state starts to develop when the minimal eigenvalue of the operator $\hat{L}$ is negative (i.e., from $\varepsilon=1-T / T_{c}$ and $\varepsilon=\Lambda+1$, we have $\left.\Lambda=-T / T_{c}\right)$. The eigenvalue $\Lambda$ also determines the minimal free energy $F$ of the giant vortex states. For the giant vortex state, we only consider the states which lie below the $F=0$ level. Inserting the solution [see Eqs. (4) and (7)] into the expression for the free energy (including the nonlinear term) and minimizing with respect to the normalization constant for the order parameter, we obtain

$$
\Psi(\rho, \varphi, z)=\left[-\Lambda \frac{\left(I_{2}\right)_{L, n}}{\left(I_{1}\right)_{L, n}}\right]^{1 / 2} \psi_{L, n}(\rho, z) \exp (i L \varphi),
$$

and the minimal value of the free energy

$$
F=-\Lambda^{2} \frac{\left(I_{2}\right)_{L, n}^{2}}{\left(I_{1}\right)_{L, n}}
$$

where
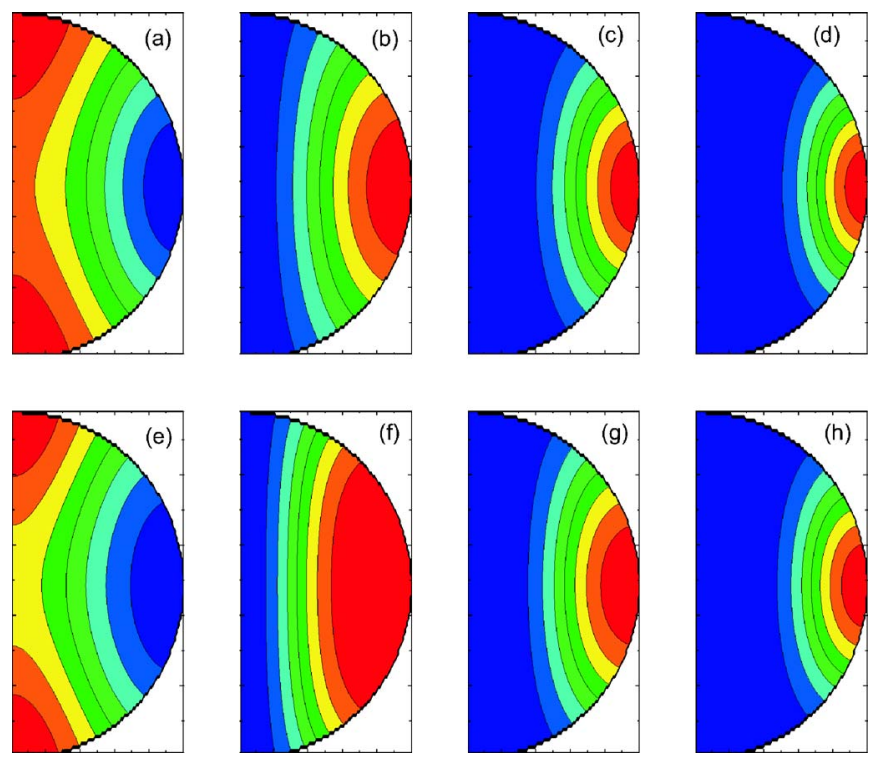

FIG. 1. (Color online) Contour plots of the Cooper-pair density in the $(\rho, z)$ plane for a sphere with radius $R=4 \xi$ for vorticity $L=0$ [(a) and (e)], $L=1$ [(b) and (f)], $L=2$ [(c) and (g)], and $L=3$ $[(\mathrm{d})$ and (h) $]$ at applied magnetic fields $H=0.18 H_{c 2}[(\mathrm{a})-(\mathrm{d})]$ and $0.42 H_{c 2}[(\mathrm{e})-(\mathrm{h})]$. The vertical axis corresponds to the direction of the applied magnetic field, i.e., the $z$ direction, while the horizontal axis corresponds to the radial direction $\rho$. High (low) Cooper-pair density is given in red (blue) regions.

$$
\begin{aligned}
& \left(I_{1}\right)_{L, n}=\int_{-R}^{R} d z \int_{0}^{\sqrt{R^{2}-z^{2}}} \rho d \rho \psi_{L, n}^{4}(\rho, z), \\
& \left(I_{2}\right)_{L, n}=\int_{-R}^{R} d z \int_{0}^{\sqrt{R^{2}-z^{2}}} \rho d \rho \psi_{L, n}^{2}(\rho, z) .
\end{aligned}
$$

As an example, we study numerically the giant vortex states for three different values of the radius $R=2,4$, and $6 \xi$. The eigenvalues and the order parameter (and therefore the Cooper-pair density and the phase of the order parameter) are obtained by solving the eigenvalue Eq. (7) for given values of the magnetic field and the vorticity $L$.

First, we show in Fig. 1 contour plots of the Cooper-pair density for a sphere with radius $R=4 \xi$, for vorticity $L=0,1$, 2 , and 3 (from left to right) at applied magnetic fields $H$ $=0.18 H_{c 2}$ (top figures) and $H=0.42 H_{c 2}$ (bottom figures). For $L=0$, no vortex is present and the Cooper-pair density is maximum around the $z$ axis. Note that the highest density is found around the poles of the sphere, while the superconducting density is suppressed near the equator. For larger $L$, magnetic field penetrates the sphere and a giant vortex is formed around the $z$ axis, which leads to $|\Psi|^{2}(\rho=0, z)=0$. Now the highest Cooper-pair density is found around the equator. With increasing vorticity, the radius of the giant vortex states increases, just like in the case of thin disks (see Ref. 1), but note that this radius tends to increase when we move away from the equator.

Next, we calculate the Cooper-pair density in the $x y$ plane at $z=0$ for the same parameters as in Figs. 1(a)-1(d). Contour plots of these results are given in Figs. 2(a)-2(d). For 

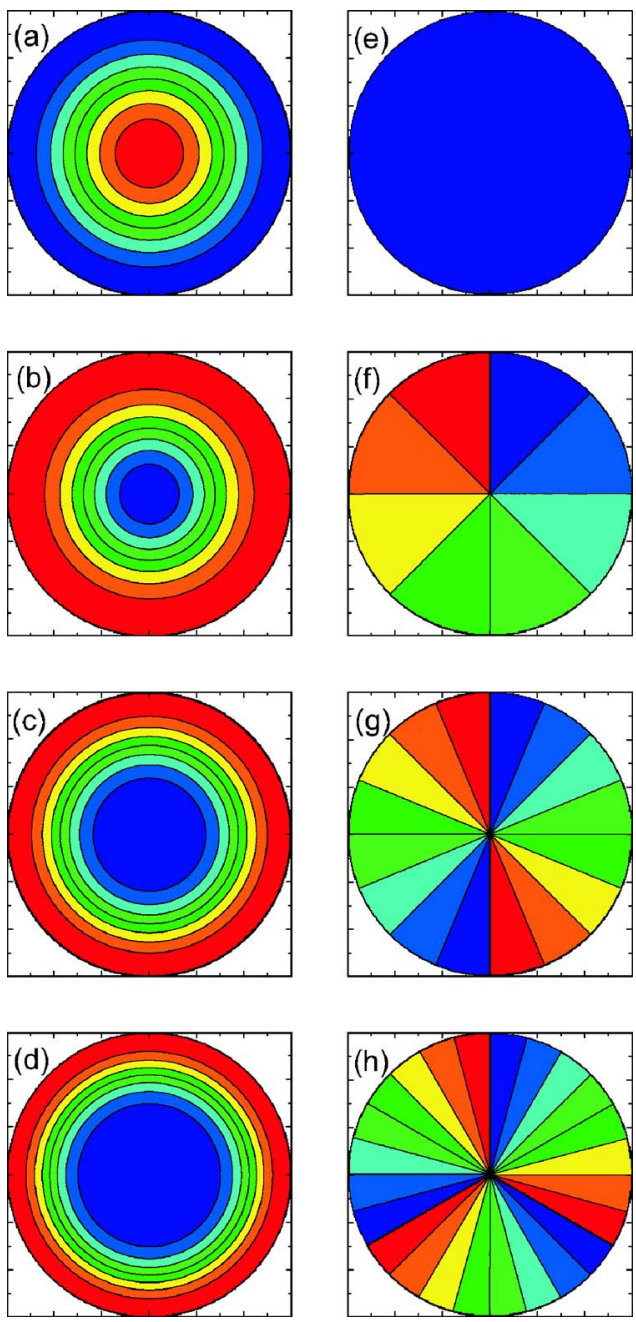

FIG. 2. (Color online) [(a)-(d)] Contour plots of the Cooper-pair density in the $x y$ plane, i.e., $z=0$, for a sphere with radius $R=4 \xi$ for vorticity $L=0,1,2$, and 3 at $H=0.18 H_{c 2}$. High (low) Cooper-pair density is given in red (blue). (e)-(h) Phase of the order parameter for the same parameters as in (a)-(d). Red (blue) indicates phases near $2 \pi(0)$.

$L=0$, the Cooper-pair density is highest in the center, while it decreases with increasing $\rho$. When $L>0$, the Cooper-pair density is zero in the center, where the giant vortex is situated. Note that from Figs. 2(a)-2(c) it is also clear that the size of the giant vortex in the center of the sphere increases with increasing $L$ as expected, because $\psi \sim \rho^{L}$ for $\rho \rightarrow 0$.

The order parameter of the giant vortex state is given by Eq. (4). The size of the order parameter, $|\psi(\rho, z)|$, can be seen from Figs. 2(a)-2(d), where $|\psi(\rho, z)|^{2}$ is given. In Figs. 2(e)-2(h), the phase of the order parameter in the $x y$ plane at $z=0$ is shown for the giant vortex states with vorticity $L=0$, 1, 2, and 3, corresponding to the situations of Figs. 2(a)-2(d). In these figures, red indicates phases near $2 \pi$ and blue indicates phases near zero. By going around the center of the vortex, the phase jumps $L$ times with $2 \pi$. From such plots we are able to determine the vorticity of the vortex state.

From the eigenvalue Eq. (7), one can obtain the eigenvalues $\Lambda$ as a function of the applied magnetic field $H$ for fixed radius $R$ and $L$. Figures 3(a)-3(c) show the eigenvalue as a
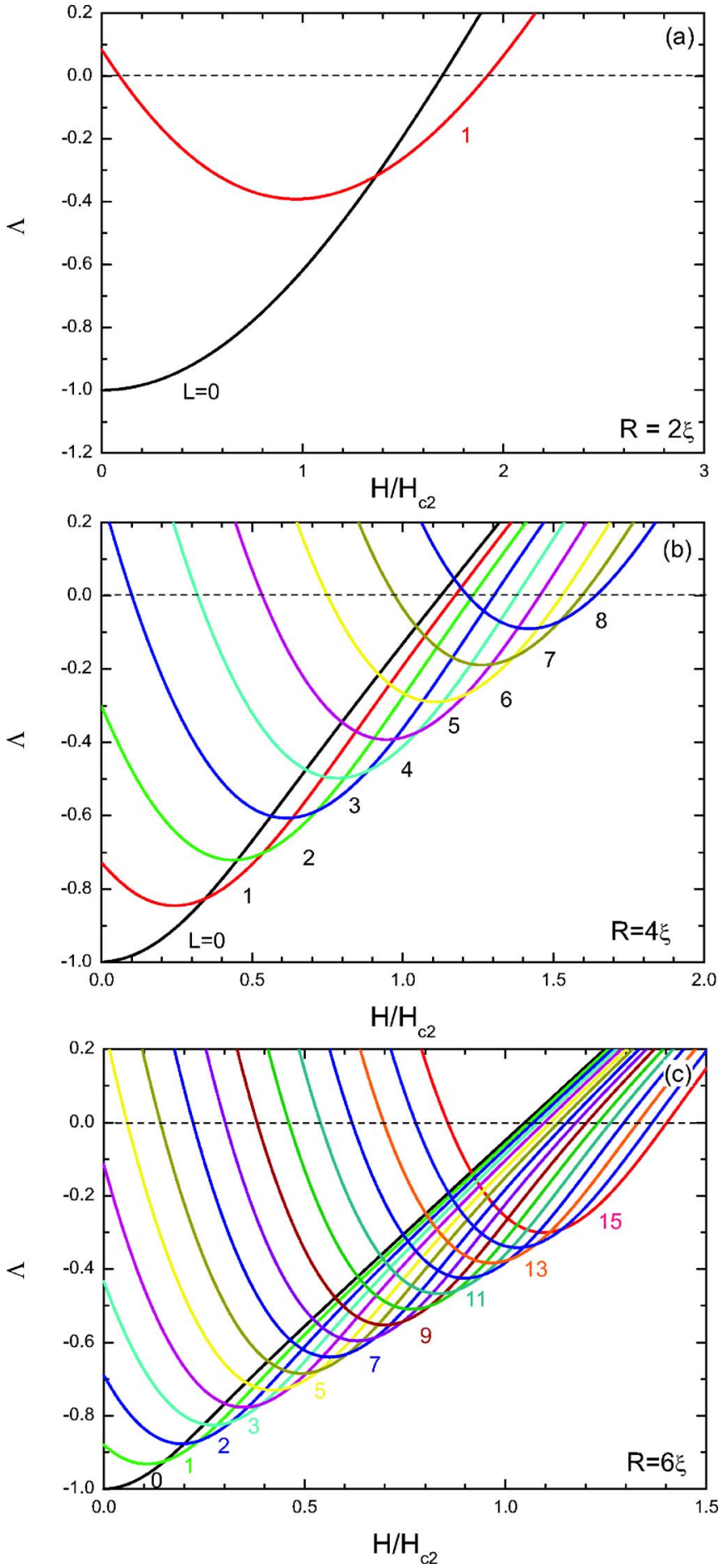

FIG. 3. (Color online) The eigenvalue $\Lambda$ as a function of the applied magnetic field $H$ for spheres with radius $R=2$ (a), 4 (b), and $6 \xi(\mathrm{c})$.

function of the applied magnetic field for spheres with radius $R=2 \xi$, $4 \xi$, and $6 \xi$, respectively. For $R=2 \xi$ and $4 \xi$, we show all (meta-)stable states, i.e., states with $\Lambda<0$ in a certain magnetic-field region. For $R=6 \xi$, we restrict ourselves to states with $L \leqslant 15$.

From Figs. 3(a)-3(c), it is clear that with increasing sphere radius, more vortex states stabilize, i.e., more vortex states have eigenvalues with $\Lambda<0$. The ground-state transi- 
tions between the different vortex states and the superconducting and/or normal transition field move to lower fields with increasing sphere radius. Notice that the thermodynamic ground state can be described by a $\Lambda=a H+b$ envelope with additional cusps near the transition fields.

From the order parameter and the eigenvalues, we can calculate the free energy $F$ as a function of the applied magnetic field for the different values of $L$ using Eqs. (9) and (10). The dependence of the free energy $F$ on the magnetic field $H$ is shown in Figs. 4(a)-4(c) for all the possible stable vortex states for spheres with radius $R=2 \xi, 4 \xi$, and $6 \xi$, respectively. The dashed horizontal line corresponds to the zero energy level. Comparing the magnetic-field dependence of the free energy for the three different sphere radii, we clearly see that with increasing radius $R$, the number of allowed vortex states increases. From Figs. 4(a)-4(c) it is also clear that with increasing radius $R$, the critical magnetic field, where $F$ becomes zero, decreases. Also, the $L \rightarrow L+1$ ground-state transition field decreases with increasing radius $R$.

Note that all free-energy curves with $L \neq 0$ start and end exactly at $F=0$. This does not mean that those vortex states are (meta-)stable over this whole region, because we restricted the space of possible solutions to the giant vortex states. Later, when considering linear combinations of different giant vortex states, we will find that the stability region of the different vortex states are reduced, in particular, near $F=0$.

Up to now, we restricted ourselves to the lowest energy state at each angular momentum. In Eq. (7) this corresponds to the situation with $n=1$, i.e., the lowest radial state or, equivalently, $\psi(\rho, z)$ has no node as a function of $\rho$. Now, we will investigate the excited states, i.e., $n>1$.

In Fig. 5, the magnetic-field dependence of the eigenvalue $\Lambda$ for both the excited and lowest states with vorticity $L=0$ and $L=1$ is given for a sphere with radius $R=2 \xi$. The red curves correspond to $L=0$ and the blue curves are for vorticity $L=1$. The solid curves are the lowest energy states for given $L$. The dashed, dash-dotted, and dotted curves correspond to the first, second, and third excited (radial) states. Since all the excited states have positive eigenvalues for all magnetic fields, it is clear that they are not stable. This is the reason why, later on when considering the order parameter as a linear combination of the $\psi_{L, n}$ states, we may restrict ourselves to $n=1$.

We show, as an example, in Figs. 6(a)-6(f) contour plots of the Cooper-pair density of the first $(n=2)$, second $(n=3)$, and third $(n=4)$ excited states for $L=0$ [Figs. 6(a) $-6(\mathrm{c})$ ] and $L=1$ [Figs. 6(d)-6(f)] at $H=0.6 H_{c 2}$. High Cooper-pair density is given by red regions, while low Cooper-pair density is given by blue regions. Regions with Cooper-pair density less than 0.01 are plotted as white. For $n=2$, we find two maxima in the Cooper-pair density located at the sphere poles, both for $L=0$ and $L=1$, while in the $z=0$ plane, the Cooper-pair density is strongly suppressed. For $n=3$, we find three maxima in both cases, and two crossing planes with low $|\Psi|$, rotated over $\theta= \pm 35^{\circ}$ with respect to the $z=0$ plane. For $n$ $=4$, we find four maxima near the sphere boundary for $L$ $=1$, but only one maximum for $L=0$ located at the sphere
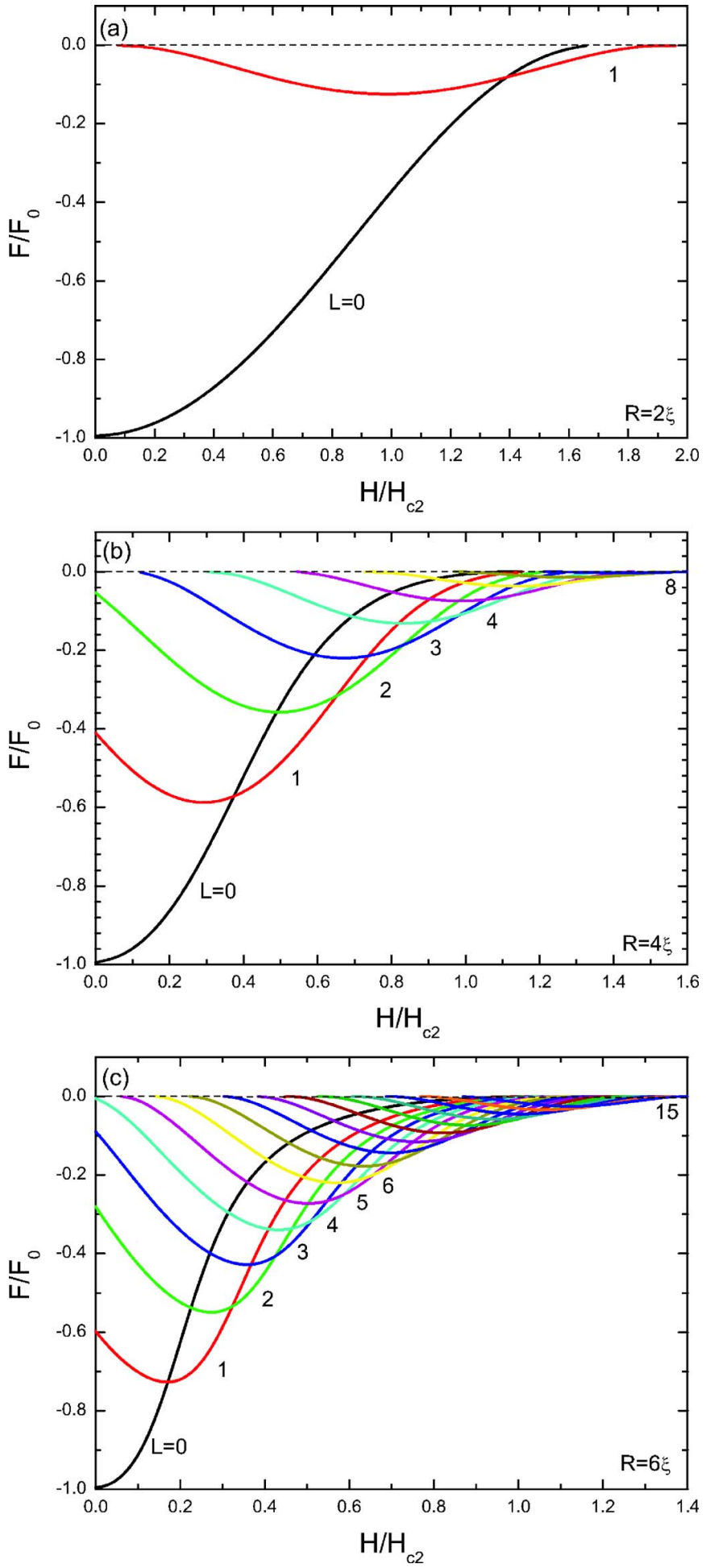

FIG. 4. (Color online) The free energy $F$ as a function of the applied magnetic field $H$ for the giant vortex states in a sphere with radius $R=2 \xi$ (a), $4 \xi$ (b), and $6 \xi$ (c).

center. For $L=1$, we find that the Cooper-pair density is suppressed in the $z=0$ plane and at two planes that are rotated over $\pm 45^{\circ}$. For $L=0$ and $n=4$, on the other hand, the Cooperpair density is zero on a shell with radius equal to $1.4 \xi$. 


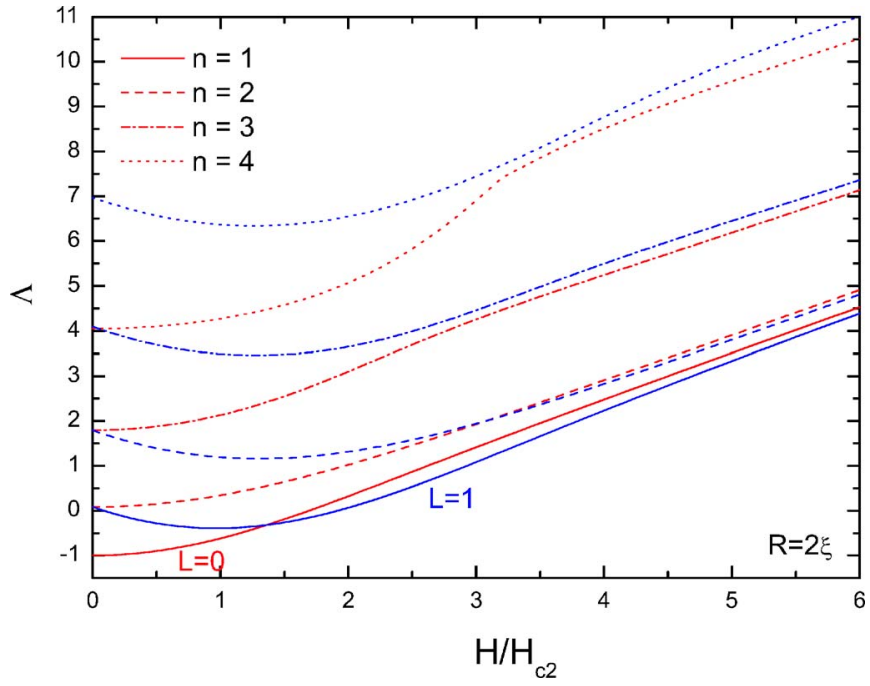

FIG. 5. (Color online) The eigenvalue $\Lambda$ as a function of the applied magnetic field for the states with $L=0$ (red curves) and $L$ $=1$ (blue curves) corresponding to the lowest eigenvalue (solid curves), the first excited state (dashed curves), the second excited state (dash-dotted curve), and the third excited state (dotted curve).

\section{MULTIVORTEX STATES}

For sufficiently large spheres, and when we move away from the $\mathrm{S} / \mathrm{N}$ boundary, the giant vortex state can break up into multivortices. In order to investigate such structures, we use the method proposed by Schweigert and Peeters, ${ }^{1,29}$ Yampolskii and Peeters, ${ }^{27}$ and Palacios ${ }^{3,28}$ for disks, and extend it here to spheres in order to determine the stability of the different multivortex configurations in spheres. Following Refs. 27 and 29, the order parameter of the multivortex
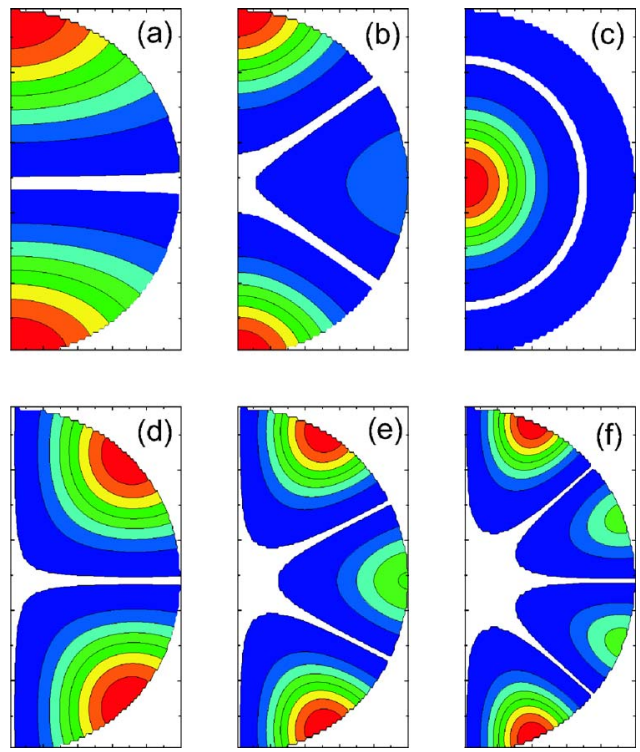

FIG. 6. (Color online) Contour plots of the Cooper-pair density of the first $(n=2)$, second $(n=3)$, and third $(n=4)$ excited states for $L=0[(\mathrm{a})-(\mathrm{c})]$ and $L=1[(\mathrm{~d})-(\mathrm{f})]$ at $H=0.6 H_{c 2}$. High (low) Cooperpair density is given in red (blue) regions. White regions indicate $|\Psi|^{2}<0.01$. state is written as a linear combination of the eigenfunctions of the linearized Ginzburg-Landau equation (1), i.e.,

$$
\Psi(\rho, \varphi, z)=\sum_{L=0}^{N} C_{L} \psi_{L}(\rho, z) \exp (i L \varphi),
$$

where the vorticity $L$ is now the value of the effective total angular momentum, which is equal to the number of vortices in the sphere. Note that we restrict ourself to $n=1$ as done in Refs. 3, 27, and 28.

Substituting Eq. (11) in the free-energy expression, we obtain $F$ as a function of the complex parameters $\left\{C_{L_{j}}\right\}$. Minimization of $F$ with respect to these parameters allows us to find the equilibrium vortex configurations and to determine their stability. The extremal points are determined by the solutions $\left\{C_{L_{j}}^{0}\right\}$ of the set of equations

$$
\frac{\partial F}{\partial C_{L_{j}}}=0 .
$$

The stable vortex states are determined by the usual criterion for a multivariable function: the matrix consisting of the second derivative (also called the Hessian matrix), i.e.,

$$
\left.\frac{\partial^{2} F}{\partial C_{L_{j}} \partial C_{L_{k}}}\right|_{C_{L_{j}}=C_{L_{j}}^{0}, C_{L_{k}}=C_{L_{k}}^{0},},
$$

must be positive definite. The giant vortices are also described by Eq. (11): they correspond to $C_{L_{j}}^{0}=0$, except for one nonzero coefficient $C_{L_{j}=L}^{0}$. This allows us to check the stability of giant vortex states with respect to transitions into a multivortex state.

Let us now consider states which are built up by only two components in Eq. (11). This restricts our analysis quantitatively but, nevertheless, will give the correct qualitative behavior for not too large values of the radius $R$, as considered in the present paper, and will facilitate the physical insight into the problem. The free energy of a two-component state is

$$
\begin{aligned}
F= & C_{L_{1}}^{4} A_{L_{1}}+C_{L_{2}}^{4} A_{L_{2}}+4 C_{L_{1}}^{2} C_{L_{2}}^{2} A_{L_{1}, L_{2}}+2 \Lambda_{L_{1}} C_{L_{1}}^{2} B_{L_{1}} \\
& +2 \Lambda_{L_{2}} C_{L_{2}}^{2} B_{L_{2}}
\end{aligned}
$$

where

$$
\begin{gathered}
A_{L_{i}}=\int_{-R}^{R} d z \int_{0}^{R(z)} \rho d \rho \psi_{L_{i}}^{4}(\rho, z), \\
B_{L_{i}}=\int_{-R}^{R} d z \int_{0}^{R(z)} \rho d \rho \psi_{L_{i}}^{2}(\rho, z), \\
A_{L_{1}, L_{2}}=\int_{-R}^{R} d z \int_{0}^{R(z)} \rho d \rho \psi_{L_{1}}^{2}(\rho, z) \psi_{L_{2}}^{2}(\rho, z),
\end{gathered}
$$

with $R(z)=\sqrt{R^{2}-z^{2}}$. Although, in general, the coefficients $C_{L_{j}}$ are complex numbers, for our two-component state, one can easily show that they are real numbers. Minimization of Eq. 
(14) with respect to $C_{L_{1}}$ and $C_{L_{2}}$ gives the possible equilibrium states:

(i) the normal state,

$$
C_{L_{1}}^{(0)}=C_{L_{2}}^{(0)}=0,
$$

(ii) the giant vortex states,

$$
\begin{aligned}
& C_{L_{1}}^{(0)}=0, \quad C_{L_{2}}^{(0)}=\left(-\Lambda_{L_{2}} \frac{B_{L_{2}}}{A_{L_{2}}}\right)^{1 / 2}, \\
& C_{L_{1}}^{(0)}=\left(-\Lambda_{L_{1}} \frac{B_{L_{1}}}{A_{L_{1}}}\right)^{1 / 2}, \quad C_{L_{2}}^{(0)}=0,
\end{aligned}
$$

(iii) the multivortex states

$$
\begin{aligned}
C_{L_{1}}^{(0)} & = \pm\left(\frac{-\Lambda_{L_{1}} A_{L_{2}} B_{L_{1}}+2 \Lambda_{L_{2}} A_{L_{1}, L_{2}} B_{L_{2}}}{A_{L_{1}} A_{L_{2}}-4 A_{L_{1,2}}^{2}}\right)^{1 / 2}, \\
C_{L_{2}}^{(0)} & = \pm\left(\frac{-\Lambda_{L_{2}} A_{L_{1}} B_{L_{2}}+2 \Lambda_{L_{1}} A_{L_{1}, L_{2}} B_{L_{1}}}{A_{L_{1}} A_{L_{2}}-4 A_{L_{1,2}}^{2}}\right)^{1 / 2} .
\end{aligned}
$$

To check the stability of the different vortex states, we have to calculate the components of the Hessian matrix (13), i.e.,

$$
\begin{gathered}
\frac{\partial^{2} F}{\partial C_{L_{1}}^{2}}=12 C_{L_{1}}^{2} A_{L_{1}}+8 C_{L_{2}}^{2} A_{L_{1}, L_{2}}+4 \Lambda_{L_{1}} B_{L_{1}}, \\
\frac{\partial^{2} F}{\partial C_{L_{2}}^{2}}=12 C_{L_{2}}^{2} A_{L_{2}}+8 C_{L_{1}}^{2} A_{L_{1}, L_{2}}+4 \Lambda_{L_{2}} B_{L_{2}}, \\
\frac{\partial^{2} F}{\partial C_{L_{1}} \partial C_{L_{2}}}=16 C_{L_{1}} C_{L_{2}} A_{L_{1}, L_{2}} .
\end{gathered}
$$

By substituting the solutions (16)-(18) into Eq. (14), we obtain the energies of the different equilibrium states, and from Eq. (19) we get the corresponding conditions for their stability.

(i) For the normal state, we obtain $F=0$. Note from Eq. (19) that for negative $\Lambda_{L_{1}\left(L_{2}\right)}$, this state is always unstable.

(ii) The energies of the giant vortex states are

$$
F_{L_{i}}=-\Lambda_{L_{i}}^{2} \frac{B_{L_{i}}^{2}}{A_{L_{i}}^{2}}
$$

which coincide with the result of Eq. (9). The conditions for the stability of the giant vortex states with vorticities $L_{1}$ and $L_{2}$ are

$$
\frac{\partial^{2} F}{\partial C_{L_{1}}^{2}}=\frac{4}{A_{L_{2}}}\left(\Lambda_{L_{1}} A_{L_{2}} B_{L_{1}}-2 \Lambda_{L_{2}} A_{L_{1}, L_{2}} B_{L_{2}}\right)>0,
$$

$$
\frac{\partial^{2} F}{\partial C_{L_{2}}^{2}}=-8 \Lambda_{L_{2}} B_{L_{2}}>0
$$

and

$$
\begin{gathered}
\frac{\partial^{2} F}{\partial C_{L_{2}}^{2}}=\frac{4}{A_{L_{1}}}\left(\Lambda_{L_{2}} A_{L_{1}} B_{L_{2}}-2 \Lambda_{L_{1}} A_{L_{1}, L_{2}} B_{L_{1}}\right)>0, \\
\frac{\partial^{2} F}{\partial C_{L_{1}}^{2}}=-8 \Lambda_{L_{1}} B_{L_{1}}>0 .
\end{gathered}
$$

(iii) The energy of the multivortex state becomes

$$
F_{L_{1}, L_{2}}=\frac{-\Lambda_{L_{1}}^{2} A_{L_{2}} B_{L_{1}}^{2}-\Lambda_{L_{2}}^{2} A_{L_{1}} B_{L_{2}}^{2}}{A_{L_{1}} A_{L_{2}}-4 A_{L_{1}, L_{2}}^{2}}+\frac{4 \Lambda_{L_{1}} \Lambda_{L_{2}} A_{L_{1}, L_{2}} B_{L_{1}} B_{L_{2}}}{A_{L_{1}} A_{L_{2}}-4 A_{L_{1}, L_{2}}^{2}},
$$

and the corresponding stability conditions are

$$
\begin{aligned}
& \frac{\partial^{2} F}{\partial C_{L_{1}}^{2}}=8 A_{L_{1}} \frac{-\Lambda_{L_{1}} A_{L_{2}} B_{L_{1}}+2 \Lambda_{L_{2}} A_{L_{1}, L_{2}} B_{L_{2}}}{A_{L_{1}} A_{L_{2}}-4 A_{L_{1}, L_{2}}^{2}}>0, \\
& \frac{\partial^{2} F}{\partial C_{L_{2}}^{2}}=8 A_{L_{2}} \frac{-\Lambda_{L_{2}} A_{L_{1}} B_{L_{2}}+2 \Lambda_{L_{1}} A_{L_{1}, L_{2}} B_{L_{1}}}{A_{L_{1}} A_{L_{2}}-4 A_{L_{1}, L_{2}}^{2}}>0,
\end{aligned}
$$

and

$$
\begin{aligned}
\frac{\partial^{2} F}{\partial C_{L_{1}}^{2}} \frac{\partial^{2} F}{\partial C_{L_{2}}^{2}}-\left(\frac{\partial^{2} F}{\partial C_{L_{1}} \partial C_{L_{2}}}\right)^{2} \\
=\left(-\Lambda_{L_{1}} A_{L_{2}} B_{L_{1}}+2 \Lambda_{L_{2}} A_{L_{1}, L_{2}} B_{L_{2}}\right) \\
\quad \times\left(-\Lambda_{L_{2}} A_{L_{1}} B_{L_{2}}+2 \Lambda_{L_{1}} A_{L_{1}, L_{2}} B_{L_{1}}\right) \\
\quad \times \frac{64}{A_{L_{1}} A_{L_{2}}-4 A_{L_{1}, L_{2}}^{2}}>0 .
\end{aligned}
$$

We will use the following notation: $L$ state for the giant vortex state with vorticity $L$, and $\left(L_{1}, L_{2}\right)$ state for the multivortex state constructed as a linear combination of the giant vortex state with vorticity $L_{1}$ and the giant vortex state with vorticity $L_{2}$. The latter state will have vorticity equal to the maximum of $L_{1}$ and $L_{2}$.

First, we calculate the free energy of all the metastable states in a superconducting sphere with radius $R=2,4$, and $6 \xi$ as a function of the applied magnetic field. This gives us the possibility to check the dependence of the stability of the multivortex state on the sphere size. Figure 7 shows the free energy for the sphere with $R=2 \xi$. Since the radius of the sphere is so small, only the 0 state, i.e., the Meissner state, and the 1 state (both giant vortex states) can nucleate. The free energy of the $L=0$ and $L=1$ states as a function of the magnetic field is exactly the same as in Fig. 4(a), but the stability region is reduced, which is determined by Eq. (21).

When we increase the sphere size, more multivortex states become stable over a certain magnetic-field region. In Fig. 8, we show the free energy of all (meta-)stable giant and multivortex states in a sphere with radius $R=4 \xi$ as a function 


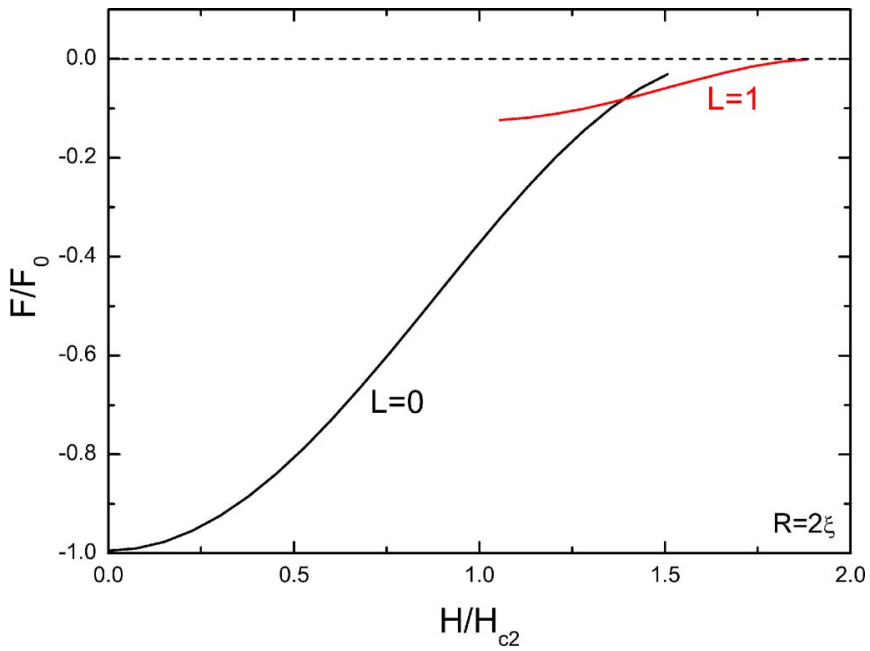

FIG. 7. (Color online) The free energy as a function of the applied magnetic field for the (meta-) stable states in a superconducting sphere with radius $R=2 \xi$.

of the applied magnetic field. The high magnetic-field region is given in more detail in Fig. 8(b). Giant vortex states are given by solid curves, $(0, L)$ multivortex states by dashed curves, $(1, L)$ multivortex states by dotted curves, and $(L$ $>1, L)$ multivortex states by dash-dotted curves. We find that giant vortex states stabilize for $L=0$ up to $L=8,(0, L)$ states for $L=2-7,(1, L)$ states for $L=4-7$, and also the $(2,7)$ and $(3,8)$ states are stable over a certain magnetic-field range. Different from the $R=2 \xi$ situation, we find that it is possible that for a given total vorticity $L$, several vortex states can nucleate at a certain magnetic field. As an example, for $L$ $=6$, we find that at $H_{0} / H_{c 2}=0.95$ two multivortex states are metastable, i.e., the $(0,6)$ state and the $(1,6)$ state, where the $(1,6)$ state has lower energy than the $(0,6)$ state. The transitions between giant vortex states with vorticity $L$ and the $(0, L)$ states are continuous (i.e., of second order) as function of the magnetic field. The other transitions, i.e., between the different multivortex states, are of first order and the derivative of the free energy exhibits a jump.

The thermodynamic ground state, i.e., the state with lowest free energy, is as follows: At low fields, the Meissner state with $L=0$ is the ground state. Around $H / H_{c 2}=0.37$, the free energy of the 0 state equals that of the 1 state, which is the ground state up to $H / H_{c 2}=0.64$ when the 2 state takes over. Note that for $L=3$ and 4 , the ground state is first given by the $(0, L)$ state and then by the giant vortex state. To show the variation of the ground state as a function of the applied magnetic field, we plot in Fig. 9 the vorticity of the ground state as a function of the field. When the ground state is a giant vortex state, the result is given by black curves, while for the $(0, L)$ state, we indicate the ground state by red curves. The top axis in Fig. 9 indicates the flux passing through the equator. As is typical for mesoscopic systems, we find that the flux increase $\Delta \Phi>\Phi_{0}$ for each $L$ value and that $L_{\text {groundstate }}$ on the average increases faster than linear with $H$.

When we further increase the sphere radius to $R=6 \xi$, the number of possible (meta)stable multivortex states increases
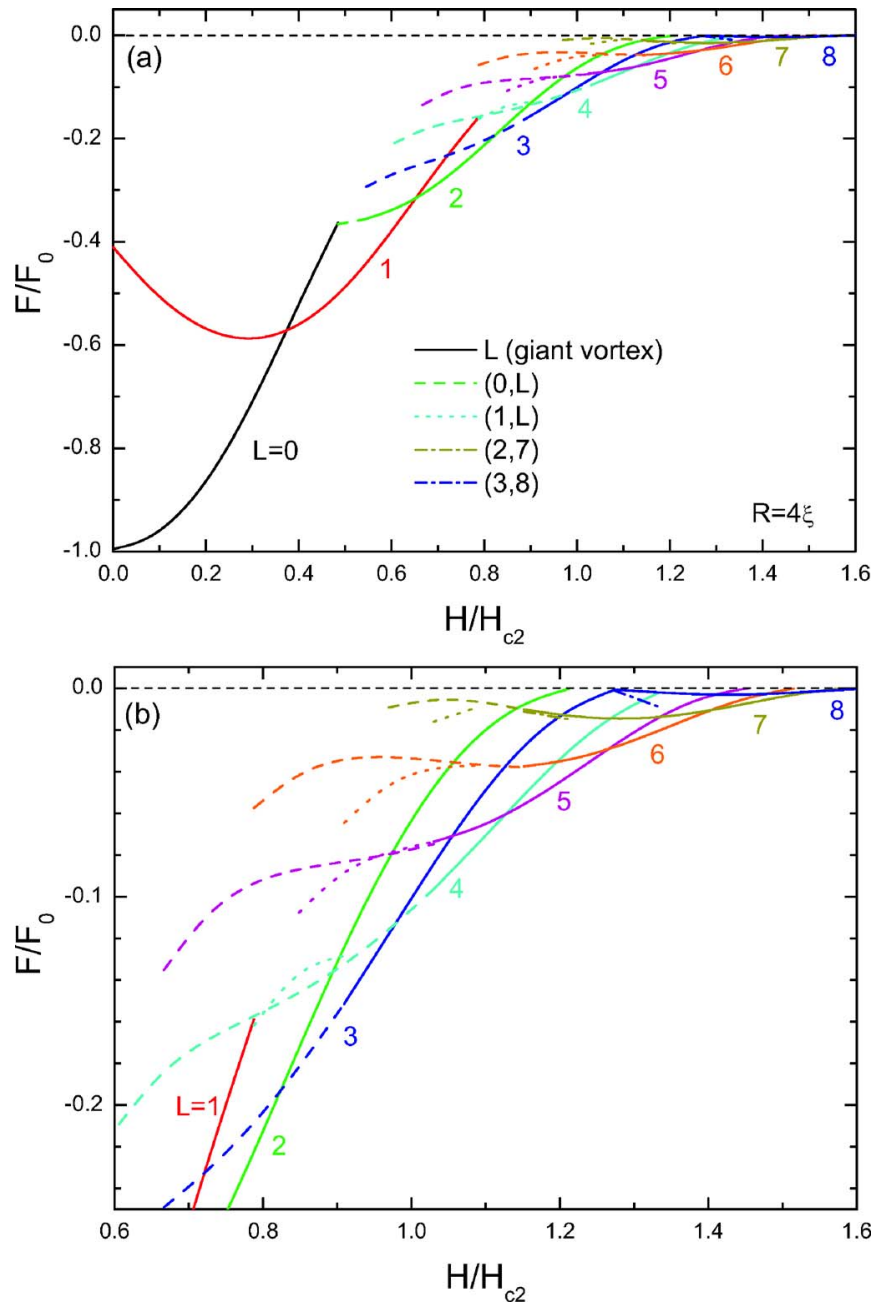

FIG. 8. (Color online) (a) The free energy as a function of the applied magnetic field for all the (meta-)stable states in a superconducting sphere with radius $R=4 \xi$. (b) The high magnetic-field region in more detail.

further. Considering only states with total vorticity $L \leqslant 15$, we find 89 states which are (meta-)stable over a certain magnetic-field region. Giant vortex states nucleate for all

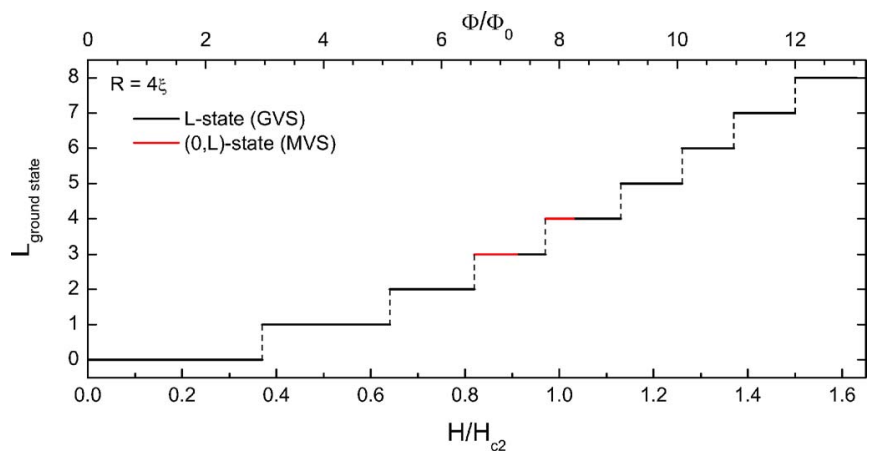

FIG. 9. (Color online) Vorticity of the ground state for a sphere with radius $R=4 \xi$ as a function of the applied magnetic field. Giant vortex states are shown in black; $(0, \mathrm{~L})$ states in red. The vertical dashed lines indicate the transition fields. The top axis gives the flux penetrating the equator. 


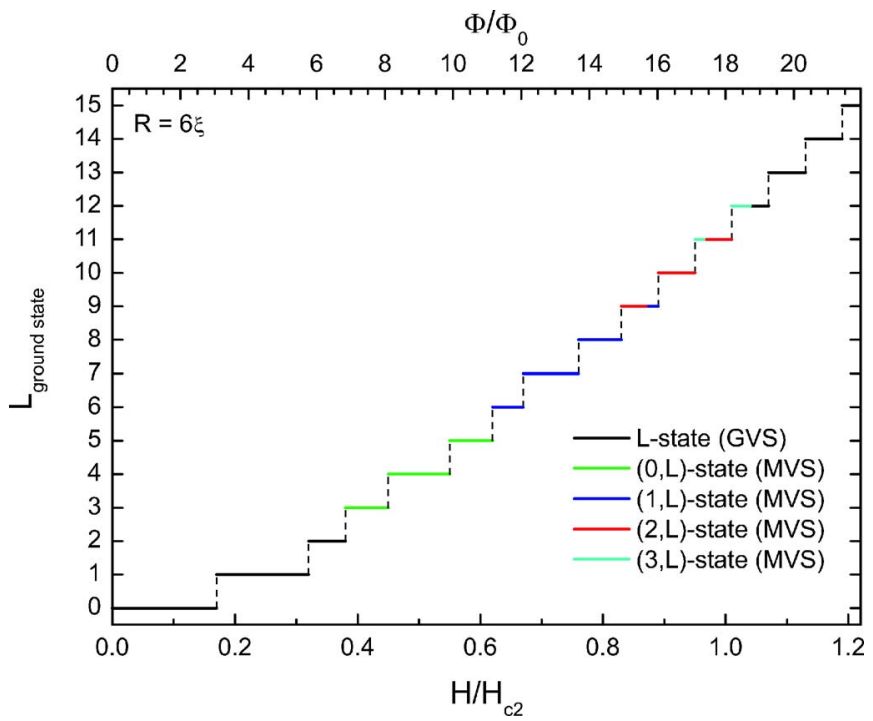

FIG. 10. (Color online) The same as Fig. 9 but now for a sphere with radius $R=6 \xi$.

values of the vorticity from $L=0$ up to $L=15$, the $(0, L)$ state for $L=2-15$, the $(1, L)$ for $L=4-15$, the $(2, L)$ for $L$ $=5-15$, the $(3, L)$ for $L=7-15$, the $(4, L)$ for $L=8-15$, the $(5, L)$ for $L=10-15$, the $(6, L)$ for $L=11-15$, the $(7, L)$ for $L=12-15$, the $(8, L)$ for $L=14$ and 15 , and finally, also the $(9,15)$ state nucleates.

Figure 10 shows the vorticity of the ground state as a function of the applied magnetic field. The different colors of the curves correspond to the different vortex states. The ground state is a giant vortex state for $L \leqslant 2$ and $L \geqslant 13$, a $(0, L)$ state for $L=3-5$, a $(1, L)$ state for $L=6-8$, and a $(2, L)$ state for $L=10$ over the whole magnetic-field region, where the state with vorticity $L$ is a ground state. For $L=9,11$, and 12 , we find a transition between two different multivortex states. For $L=9$, the ground state is first the $(2,9)$ state and transits with increasing field to the $(1,9)$ state. Note that this transition is of first order. For $L=11$, the $(3,11)$ state transits into the $(2,11)$ state with increasing field, while for $L=12$, the $(3,12)$ state transits into the giant vortex state with $L=12$. For $L \geqslant 13$, only giant vortex states are found, which is a manifestation of surface superconductivity at high magnetic fields.

To gain further insight into the nature of the vortex state, we investigate the Cooper-pair density in the $x y$ plane of the sphere. In Fig. 11, contour plots of the Cooper-pair density in the $x y$ plane are shown for a sphere with radius $R=4 \xi$. Figures $11(\mathrm{a})-11(\mathrm{~d})$ correspond to the $(0,3),(0,4),(0,5)$, and $(0,6)$ states at a magnetic field $H=0.85 H_{c 2}$. High Cooper-pair density is given by red regions and low Cooper-pair density by blue regions. The white point is the center of the vortex where the Cooper-pair density $|\Psi|^{2}<0.001$. When we combine a giant vortex state with $L=0$ with a giant vortex state with $L=L_{1}>0$, we find that the vortex state consists of $L_{1}$ vortices located on a shell, while there is no vortex in the center.

What happens when we take a linear combination of two giant vortex states with $L>0$ ? Let us first consider the $\left(1, L_{1}\right)$ states, i.e., a linear combination of the $L=1$ giant
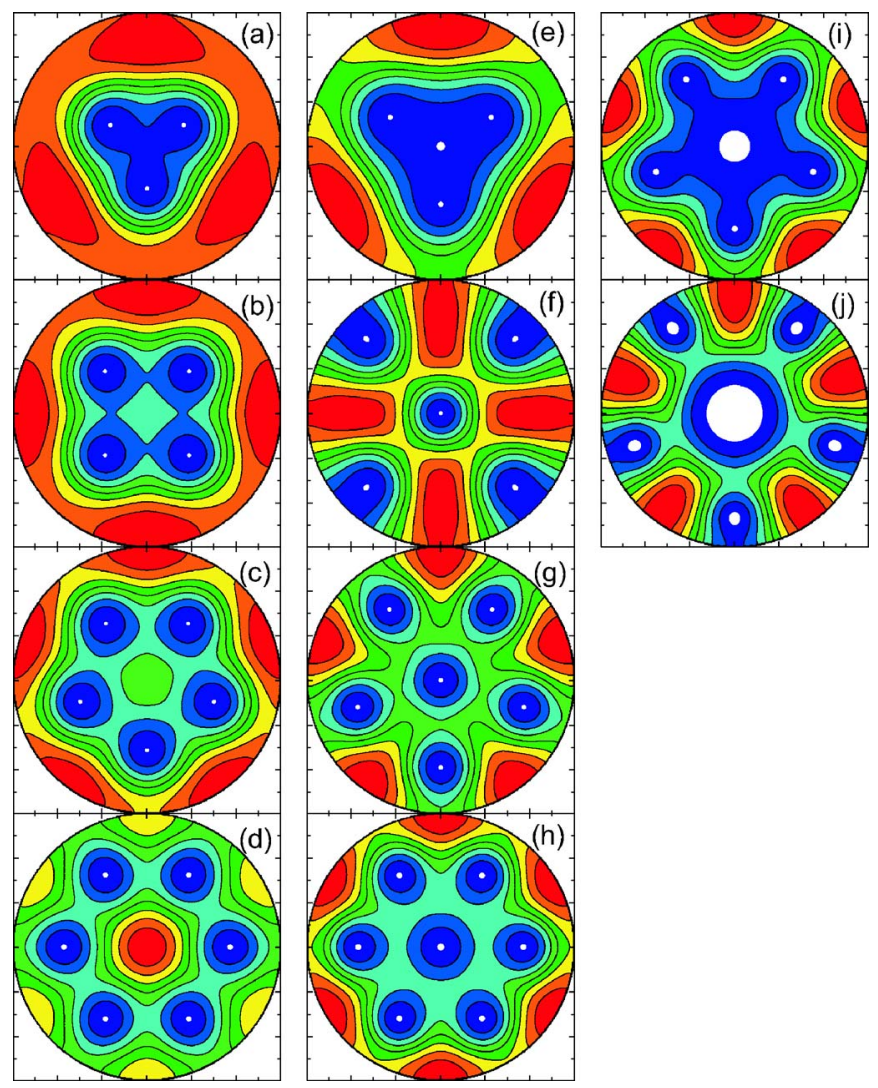

FIG. 11. (Color online) Contour plots of the Cooper-pair density in the $z=0$ plane of a sphere with radius $R=4 \xi$ for the $(0,3),(0,4)$, $(0,5)$, and $(0,6)$ states at $H=0.85 H_{c 2}[(\mathrm{a})-(\mathrm{d})]$; the $(1,4),(1,5),(1,6)$, and $(1,7)$ states at $H / H_{c 2}=0.85,0.90,0.95$, and 1.05 [(e)-(h)], respectively; and the $(2,7)$ and $(3,8)$ states at $H=1.1 H_{c 2}[(i)$ and $(j)]$. High (low) Cooper-pair density is given in red (blue and white).

vortex state and a giant vortex state with vorticity $L_{1}$. In Figs. 11(e)-11(h), the Cooper-pair density for a sphere with $R$ $=4 \xi$ is shown for $z=0$ for the $(1,4)$, the $(1,5)$, the $(1,6)$, and the $(1,7)$ combinations at $H / H_{c 2}=0.38,0.42,0.79$, and 0.87 , respectively. From Fig. 8 we know that the magnetic-field ranges over which these $\left(1, L_{1}\right)$ states are stable are much smaller than for the $\left(0, L_{1}\right)$ states. The $\left(1, L_{1}\right)$ states only exist in very narrow magnetic-field regions. From Figs. 11(e)-11(h) we notice that there is one vortex in the center while $L_{1}-1$ vortices are situated on a shell around this central vortex.

Next, we try to find stable linear combinations of two giant vortex states with $L>1$ for $R=4 \xi$. We find that only two of these states are stable, i.e., the $(2,7)$ state and the $(3,8)$ state. The Cooper-pair density of these multivortex states are shown in Figs. 11(i) and 11(j) at $H=1.1 H_{c 2}$. In both cases, the multivortex state consists of a giant vortex in the center surrounded by a shell of several single vortices. For the $(2,7)$ state, the giant vortex has vorticity $L=2$, while for the $(3,8)$ state, the central giant vortex has vorticity $L=3$. In both cases, the giant vortex is encircled by a shell of five single vortices.

With increasing sphere radius, the $\left(L_{1}, L_{2}\right)$ multivortex stabilizes for many more values of $L_{1}$ and $L_{2}$. For spheres with radius $R=6 \xi$, we find that multivortex states up to the 

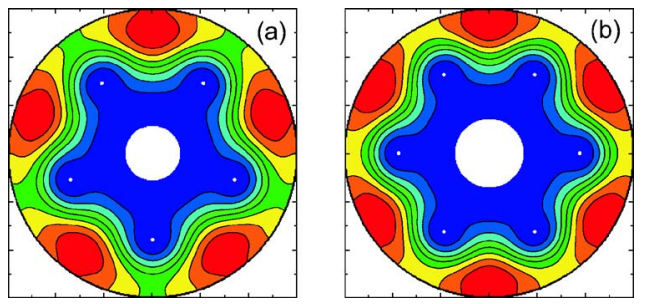

FIG. 12. (Color online) Contour plots of the Cooper-pair density in the $x y$ plane of a sphere with radius $R=6 \xi$ for the $(4,9)$ and $(5,11)$ states.

combination of $L_{1}=9$ and $L_{2}=15$ can be stabilized. As an example, we show in Figs. 12(a) and 12(b) contour plots of the $(4,9)$ and $(5,11)$ states in a sphere with radius $R=6 \xi$. The $(4,9)$ state consists of a central giant vortex with vorticity $L=4$, encircled by five single vortices, and the $(5,11)$ state consists of a giant vortex with vorticity 5 in the center and six single vortices on a shell around this vortex.

Up to now, we gave examples of stable multivortex states at a fixed magnetic field. But what is the influence of the magnetic field on the position of the vortices? To show this effect, we plot in Figs. 13(a)-13(d) the $(0,4)$ state in a sphere with radius $R=4 \xi$ at several values of the applied magnetic field, i.e., $H=0.61 H_{c 2}, H=0.73 H_{c 2}, H=0.85 H_{c 2}$, and $H$ $=1.03 H_{c 2}$, respectively. With increasing magnetic field, the vortices clearly move in the direction of the center. Note that in Fig. 13(d) the vortices of the $(0,4)$ state are close to the center, where we have an almost circular area where $|\Psi|^{2}$ is very small. When we further increase the applied magnetic field, the four vortices combine into one big giant vortex with vorticity $L=4$, i.e., the $(0,4)$ multivortex state transits into a $L=4$ giant vortex state. This transition is continuous, i.e., of second order.

When encircling a single vortex, the phase of the order parameter changes with $2 \pi$. For giant vortex states, the phase of the order parameter will change by $L \times 2 \pi$ when encircling the giant vortex. Now, we investigate the phase of the order parameter in case of a multivortex state. In Figs.
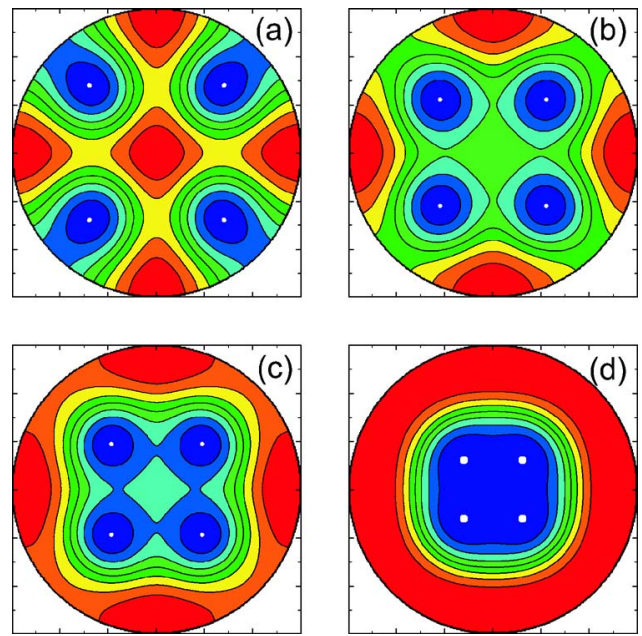

FIG. 13. (Color online) Contour plots of the Cooper-pair density in the $x y$ plane of a sphere with radius $R=4 \xi$ for the $(0,4)$ state at $H / H_{c 2}=0.61,0.73,0.85$, and 1.03 .
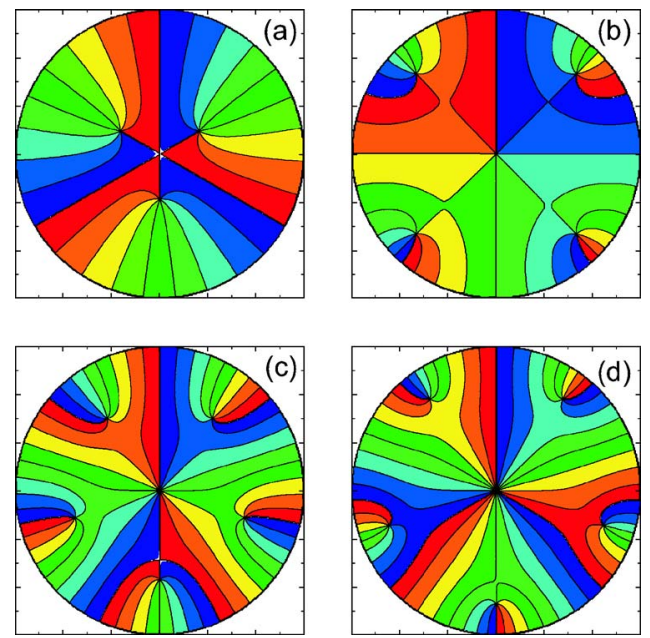

FIG. 14. (Color online) Contour plots of the phase of the order parameter in the equator plane of the sphere $(R=4 \xi)$ for the $(0,3)$, $(1,5),(2,7)$, and $(3,8)$ states at $H / H_{c 2}=0.65,0.65,1.15$, and 1.27 , respectively.

14(a)-14(d), the phase of the order parameter in the $z=0$ plane is shown for the $(0,3),(1,5),(2,7)$, and $(3,8)$ states in a sphere with radius $R=4 \xi$ at magnetic fields $H / H_{c 2}=0.65$, $0.65,1.15$, and 1.27 , respectively. Note that these multivortex states are (meta-)stable at the considered magnetic-field values. Red regions correspond to phases near $2 \pi$, while blue regions correspond to phases near zero. Let us first consider the $(0,3)$ state in Fig. 14(a). When encircling the sphere near the boundary, we find that the phase changes three times with $2 \pi$. This means that the total vorticity is 3 . When encircling the center of the sphere, the phase remains almost zero (or $2 \pi$, which means the same). So, there is no vortex in the center. The three vortices are clearly on a shell around the center of the sphere. When encircling a single vortex, the phase changes clearly by $2 \pi$, which means that the vortex is singly quantized. From Fig. 14(b) we know that the $(1,5)$ state has total vorticity 5 . It contains one singly quantized vortex in the center and four single vortices on a shell. The $(2,7)$ state [Fig. $14(\mathrm{c})]$ has total vorticity 7 , a giant vortex with vorticity $L=2$ in the center and five singly quantized vortices on a shell. Fig. 14(d) shows the $(3,8)$ state, which consists of a giant vortex with $L=3$ in the center and five single vortices on a shell. The total vorticity is 8 .

For disks, the stability of the multivortex state was investigated by Schweigert et al. ${ }^{2,29}$ Palacios, ${ }^{3,28}$ and Yampolskii and Peeters. ${ }^{27}$ Above, we adapted this stability criterion to superconducting spheres. For disks, we know that the coefficients $C_{L}$ are usually complex numbers, but for the twocomponent state it was shown that the $C_{L}$ are real numbers, and the stable states have a positive Hessian matrix. This criterion also works well for the two-component situation in spheres. But in this case, the coefficients are complex even in the two-component situation. However, for the stable states, we found that the coefficients $C_{L}$ are real and positive. We checked this for all the vortex states studied in our work. This result can be used as a more simple criterion to 


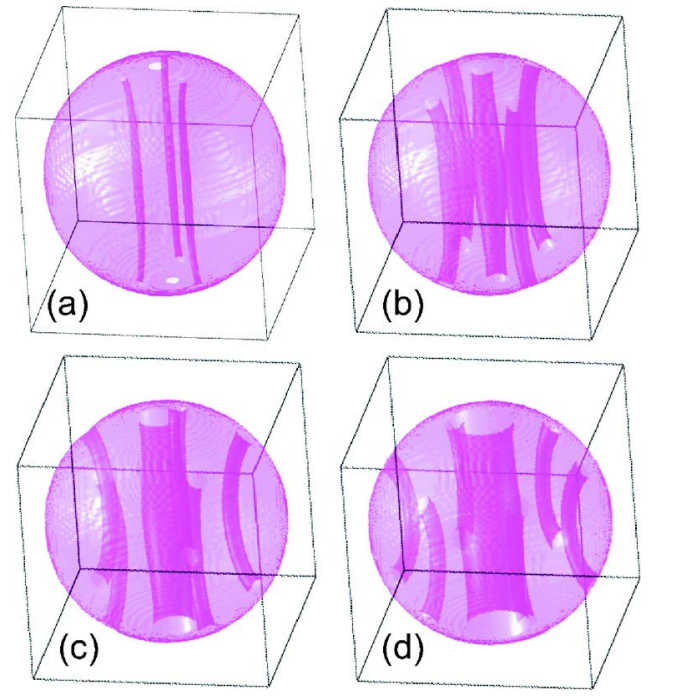

FIG. 15. (Color online) Three-dimensional figures of the $(0,3)$, $(1,5),(2,7)$, and $(3,9)$ states in a superconducting sphere with radius $R=4 \xi$.

determine the stability of the multivortex states in mesoscopic spheres.

Up to now, we focused on the Cooper-pair density and the order parameter of the multivortex state, i.e.,

$$
\Psi(\rho, \varphi, z)=C_{L_{1}} \psi_{L_{1}}(\rho, z) e^{i L_{1} \varphi}+C_{L_{2}} \psi_{L_{2}}(\rho, z) e^{i L_{2} \varphi}
$$

in the equator plane of the sphere. Due to the boundary of the sphere, it can be expected that the spatial distribution of the vortices will change when going away from the equator plane, i.e., when considering $z \neq 0$. As an example, we show in Figs. 15(a)-15(d) the three-dimensional distribution of the Cooper-pair density in a superconducting sphere with radius $R=6 \xi$ at $H=0.70 H_{c 2}$ for the $(0,3),(1,5),(2,7)$, and $(3,9)$ states. Note that the diameters of the vortices in these figures are different from the vortices in the two-dimensional contour plots above. Here we show the isosurfaces where the Cooper-pair density has a fixed but low value. Therefore, we only see the sphere boundary and the "boundaries" of the vortices. Note that the vortices bend toward the boundary of the sphere when moving away from the $z=0$ plane, except for the (giant) vortex in the center. Figure 15(a) shows the $(0,3)$ state, where three vortices are on a shell in the $z=0$ plane. It is shown that all vortices bend toward the outer boundary with increasing $z$. Figure $15(\mathrm{~b})$ shows the $(1,5)$ state, where one vortex is in the center, which is surrounded by four vortices on a shell. The central vortex stays along the $\rho=0$ axis, while the other vortices bend outward. Thus, for $z \approx R$, only the central vortex is inside the sphere, while the other vortices left the sphere. Figures 15(c) and 15(d) show the $(2,7)$ and $(3,9)$ states, where a central giant vortex is surrounded by single vortices. For $z>0$, the giant vortex remains around the $z$ axis, while the other vortices move toward the outer boundary. From these figures, it is also clear that the radius of the giant vortex increases with vorticity $L$.

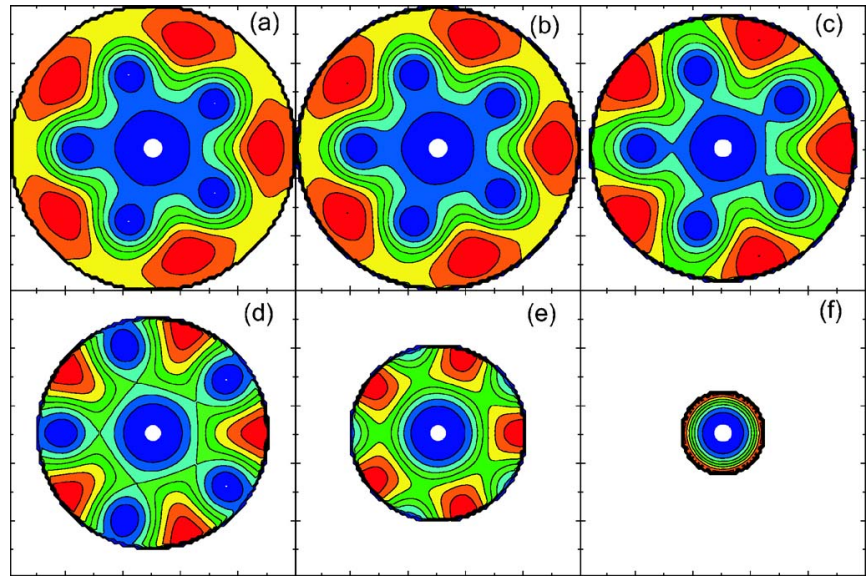

FIG. 16. (Color online) Contour plots of the Cooper-pair density in the $x y$ plane for the configuration corresponding to Fig. 15(c) for different values of $z$, i.e., $z / \xi=0,1.2,2.4,3.6,4.8$, and 5.9.

Note further that the radius of the giant vortex is larger around $z=0$ and decreases slightly when we move away from the equator plane.

To show in more detail the $z$ dependence of the Cooperpair density, we plot in Fig. 16 different two-dimensional cuts of Fig. 15(c) at $z / \xi=0,1.2,2.4,3.6,4.8$, and 5.9, respectively. For $z=0$, we find a giant vortex with $L=2$ in the center surrounded by five vortices. With increasing $z$, these five vortices move toward the outer boundary [see Figs. 16(b) $-16(d)]$. Around $z=4.8 \xi$ [see Fig. 16(e)], they leave the superconducting sphere. When we further increase $z$, we only find the central giant vortex [see Fig. 16(f)].

\section{CONCLUSIONS}

We studied the possible (meta-)stable vortex states in superconducting spheres with radius $R=2,4$, and $6 \xi$ in the presence of an external applied magnetic field. First, we limited our study to the axial symmetric giant vortex states by treating the first linear Ginzburg-Landau equation as an eigenvalue problem. We found that with increasing sphere radius, more vortex states stabilize, while the superconducting and/or normal transition field decreases. Also, the number of (meta-)stable vortex states at a given magnetic field increases when the radius increases. Although not stable for the considered spheres, we also investigated higher-energy states, where $\Psi(\vec{r})$ has a nodal plane.

Second, we took linear combinations of the different giant vortex states in order to find approximate solutions of the nonlinear Ginzburg-Landau theory. In this way, we can also find (meta-)stable multivortex state solutions and determine the stability regions of the different giant vortex states. With increasing sphere radius, the number of (meta-)stable multivortex states and their stability region increase. We found second-order transitions between the $(0, L)$ multivortex and giant vortex states with vorticity $L$, while the transitions between the different multivortex states with the same vorticity 
and transitions between states with different vorticities are of first order. From the three-dimensional structure of the vortices, we find that the central (giant) vortex stays around the $z$ axis, when we move away from the equator plane, and the size of the giant vortex slightly decreases. The single vortices (situated on a shell around the $z$ axis) move toward the sphere boundary with increasing $|z|$.

\section{ACKNOWLEDGMENTS}

This work was supported by the Flemish Science Foundation (FWO-Vl), the Belgian Science policy, and the ESF-network ADQJJ. B.J.B. acknowledges support from FWO-Vl. Discussions with S. Yampolskii are gratefully acknowledged.
*Electronic address: francois.peeters@ua.ac.be

${ }^{1}$ V. A. Schweigert and F. M. Peeters, Phys. Rev. B 57, 13817 (1998).

${ }^{2}$ V. A. Schweigert, F. M. Peeters, and P. S. Deo, Phys. Rev. Lett. 81, 2783 (1998); P. S. Deo, V. A. Schweigert, F. M. Peeters, and A. K. Geim, ibid. 79, 4653 (1997).

${ }^{3}$ J. J. Palacios, Phys. Rev. B 58, R5948 (1998).

${ }^{4}$ J. J. Palacios, Phys. Rev. Lett. 84, 1796 (2000).

${ }^{5}$ B. J. Baelus, L. R. E. Cabral, and F. M. Peeters, Phys. Rev. B 69, 064506 (2004).

${ }^{6}$ L. R. E. Cabral, B. J. Baelus, and F. M. Peeters, Phys. Rev. B 70, 144523 (2004).

${ }^{7}$ V. V. Moshchalkov, L. Gielen, C. Strunk, R. Jonckheere, X. Qiu, C. Van Haesendonck, and Y. Bruynseraede, Nature (London) 373, 319 (1995).

${ }^{8}$ V. Bruyndoncx, L. Van Look, M. Verschuere, and V. V. Moshchalkov, Phys. Rev. B 60, 10468 (1999).

${ }^{9}$ A. K. Geim, I. V. Grigorieva, S. V. Dubonos, J. G. S. Lok, J. C. Maan, A. E. Filippov, and F. M. Peeters, Nature (London) 390, 259 (1997).

${ }^{10}$ A. K. Geim, S. V. Dubonos, I. V. Grigorieva, K. S. Novoselov, F. M. Peeters, and V. A. Schweigert, Nature (London) 407, 55 (2000).

${ }^{11}$ A. Kanda, M. C. Geisler, K. Ishibashi, Y. Aoyagi and T. Sugano, in Quantum Coherence and Decoherence ISQM-Tokyo '98, edited by Y. A. Ono and K. Fujikawa (Elsevier, Amsterdam, 1999), p. 229; A. Kanda and Y. Ootuka, Microelectron. Eng. 63, 313 (2002); A. Kanda and Y. Ootuka, Physica C 404, 205 (2004).

${ }^{12}$ A. Kanda, B. J. Baelus, F. M. Peeters, K. Kadowaki, and Y. Ootuka, Phys. Rev. Lett. 93, 257002 (2004).

${ }^{13}$ B. J. Baelus, A. Kanda, F. M. Peeters, Y. Ootuka, and K. Kadowaki, Phys. Rev. B 71, 140502(R) (2005).
${ }^{14}$ L. F. Chibotaru, A. Ceulemans, V. Bruyndoncx, and V. V. Moshchalkov, Nature (London) 408, 833 (2000); Phys. Rev. Lett. 86, 1323 (2001).

15 J. Bonča and V. V. Kabanov, Phys. Rev. B 65, 012509 (2001); T. Mertelj and V. V. Kabanov, ibid. 67, 134527 (2003).

${ }^{16}$ B. J. Baelus and F. M. Peeters, Phys. Rev. B 65, 104515 (2002).

${ }^{17}$ A. S. Mel'nikov, I. M. Nefedov, D. A. Ryzhov, I. A. Shereshevskii, V. M. Vinokur, and P. P. Vysheslavtsev, Phys. Rev. B 65, 140503(R) (2002).

${ }^{18}$ V. R. Misko, V. M. Fomin, J. T. Devreese, and V. V. Moshchalkov, Phys. Rev. Lett. 90, 147003 (2002).

${ }^{19}$ M. Morelle, G. Teniers, L. F. Chibotaru, A. Ceulemans, and V. V. Moshchalkov, Physica C 369, 351 (2002).

${ }^{20}$ M. Morelle, J. Bekaert, and V. V. Moshchalkov, Phys. Rev. B 70, 094503 (2004)

${ }^{21}$ B. J. Baelus, A. Kanda, N. Shimizu, K. Tadano, Y. Ootuka, K. Kadowaki, and F. M. Peeters, Phys. Rev. B 73, 024514 (2006).

${ }^{22}$ A. F. Slachmuylders, B. Partoens, and F. M. Peeters, Phys. Rev. B 71, 245405 (2005).

${ }^{23}$ R. Geurts, M. V. Milosevic, and F. M. Peeters, Phys. Rev. Lett. 97, 137002 (2007).

${ }^{24}$ G. F. Zharkov, V. G. Zharkov, and A. Yu. Zvetkov, Phys. Rev. B 61, 12293 (2000).

${ }^{25}$ Q. Du, J. Math. Phys. 46, 095109 (2005); Q. Du and L. Ju, J. Comput. Phys. 201, 511 (2004).

${ }^{26}$ A. K. Azamat, D. Y. Vodolazov, and F. M. Peeters, Europhys. Lett. 74, 151 (2006).

${ }^{27}$ S. V. Yampolskii and F. M. Peeters, Phys. Rev. B 62, 9663 (2000).

${ }^{28}$ J. J. Palacios, Physica B 256-258, 610 (1998).

${ }^{29}$ V. A. Schweigert and F. M. Peeters, Phys. Rev. Lett. 83, 2409 (1999). 